\title{
Communication and Decision Support Systems
}

Luigi Palestini ${ }^{1,2}$

${ }^{1}$ Italian Fire and Rescue Service, Rome 00184, Italy

${ }^{2}$ Master Course in Protection Against CBRNe Events, University of Rome “Tor Vergata”, Rome 00133, Italy

Corresponding Author Email: luigi.palestini@ vigilfuoco.it

https://doi.org/10.18280/ijsse.110413

Received: 9 January 2021

Accepted: 12 July 2021

\section{Keywords:}

common alerting protocol, communication systems, DSS, emergency management, GIS,

ITC, situation assessment

\begin{abstract}
In emergencies, assessment and communication activities are particularly important for the support of the top decision-making bodies, to evaluate "just in time" the best actions to be taken. The multiple problems to be solved require specific skills in different areas. Upon the occurrence of a calamity, the authorities must answer questions such as "is a given place safe from the threat (e.g., an oncoming flood)?", that's why today knowledge of tools that can support decision-making is increasingly necessary: the so-called Decision Support Systems (DSS), software that allow users to improve situation assessment, helping all those who must make strategic decisions. Hand in hand with the growing interest in DSS there is an increasing use of communication systems based on IT. First responders know that to face an emergency everything must be prepared and planned, also communication. In fact, DSS and voice/data transmission systems are often integrated into a single system, as proposed by the European projects FIRE IN and IN PREP, because managing information is crucial for carrying out rescue activities in the best possible way. This work describes the impact of new technologies on rescue and emergency management in Italy and Europe, highlighting the challenges associated with their use.
\end{abstract}

\section{INTRODUCTION}

An emergency can be defined as any deviation from normal operating conditions, such as to determine situations of possible damage to people or things. In Italy, those who mainly deal with emergency management are firefighters (Fire and Rescue Service) [1] and Civil Protection Department. The Italian Fire and Rescue Service, in order to look after the safety of people and preservation of property, ensures the technical measures characterized by the requirement of immediacy performance, for which technical skills are required (even if highly specialized teams and appropriate skills are required) and for the same purpose it carries out studies and experimental tests in the specific sector. The Italian Fire and Rescue Service urgent technical rescue activity includes:

(1) rescue activity in the event of fires, uncontrolled releases of energy, structural collapses, landslides, floods, or other public calamities.

(2) contrasting risks from nuclear energy and the use of biological, chemical and radiological substances.

The relevant literature and, above all, the experience gained in the field, tell us that the two characteristics of a crisis, compared to periods of quiet, are the need to make decisions quickly and (often) in the absence of all the information that would be needed and the emotional stress that derives from this time pressure and from having to make decisions, without the certainty that they are the correct ones.

To overcome these difficulties, the organizations that have been dealing with rescue and civil protection for several years have shown interest in technologies that can be useful for the management of emergencies.

This work describes decision support and voice/data communication systems, technologies and resources in use by the Italian Fire and Rescue Service and commonly used in case of disasters, to respond to the needs of strategic assessment of scenarios and emergency management. It also discusses its strengths and weaknesses, which will represent important challenges for the future.

\section{DECISION SUPPORT AND INTEROPERABILITY}

\subsection{Incident command system}

An emergency is any deviation from normal operational conditions, such as to determine situations of possible damage to men or things. In this context the Italian Fire and Rescue Service plays a fundamental role, ensuring urgent technical rescue interventions, securing places, structures, systems and giving assistance to the population, immediately taking charge of the technical and operational direction of rescue activities.

In an emergency, or rather more generally, in crisis situations, needs, priorities, hierarchies, structures involved, tools and the necessary skills are profoundly different and require the adoption of an organization different from that used in "ordinary conditions".

In simple or complex emergency interventions, for the Italian firefighters the chain of command operates on three levels:

(1) strategic level, which consists of a long-term action plan used to set up and subsequently coordinate the actions aimed at achieving the complete management of the emergency and achieving the objectives set.

(2) tactical level, that is a set of plans and means used to 
obtain the expected results for specific areas and tasks.

(3) operational level, which is implemented by the teams and includes the carrying out of maneuvers necessary to obtain the expected results at a tactical level.

The heart of the activities and the decision-making center is made up of the local and regional operations rooms (including the advanced command posts, often consisting of equipped vans) and the National Operations Center.

The organizational support tool in emergencies adopted by the firefighters at a strategic, tactical and operational level is the Incident Command System, commonly known as ICS (see Figure 1). It can be defined as a systematic tool, used for command, control and coordination of emergency interventions, or, more specifically, as the set of personnel, policies, procedures, structures and equipment, integrated into a common organization and designed to improve emergency response operations of all types and complexity.

The structure of this model is simple: there is one top coordination figure, the Commander of the accident, who manages four line functions (Operational, Planning, Logistics and Administration) and three staff functions (Security, Liaison function and Communication). The ICS includes the procedures for its implementation and the criteria for defining the hierarchical chain of command for the control of funds, personnel, facilities, equipment and communications. ICS is certainly the best-known organizational model for emergency management and is used internationally; it is recognized by numerous public and private organizations, including NATO, ICAO and NFPA and is part of The National Interdepartmental Approach to Incident Management (NIIMS) implemented in the United States of America [1].

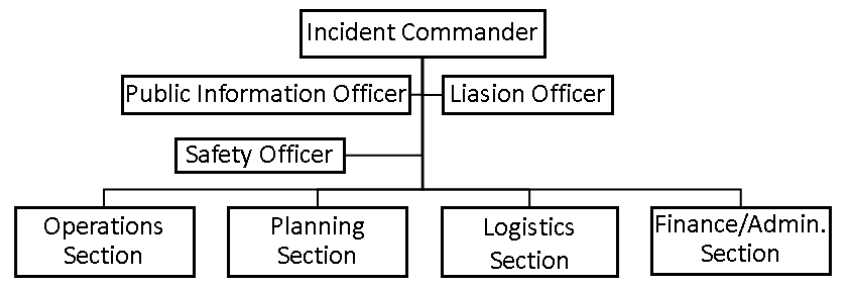

Figure 1. The incident command system

\subsection{Topography applied to rescue}

Topography applied to rescue (TAS) supports firefighter's activities, using advanced geographic information, related to the emergency scenario and it is one of the first decision support tools that the Italian firefighters have equipped themselves with. The TAS service use a Geographic Information System (GIS), for working with maps and geographic information, share data about emergency scenarios (as position of resources and interventions) and as a Spatial Decision Support System SDSS. Firefighters have always used cartography. When a firefighters team goes to make an emergency intervention, not knowing the way to go, they use a road map, if in the city, or a topographical card, if out of town. Through various computer programs of cartographic management, today it is possible to view the operations area on the screen. The management of interventions from the operations room and from the forward command post is therefore considerably facilitated. This can be decisive in the most varied interventions: search for missing people, forest fires, NBCR scenarios, aquatic rescue, floods, earthquakes, landslides, interventions carried out by helicopter, interventions in industrial sites at risk, etc. Also, through the computer it is also possible to manage GPS devices in the field, to check the position of the operating units at any time and to proceed with order and rationality in carrying out rescue operations. The operational experience gained in recent years has allowed us to understand how the TAS has a transversal value in all the activities of firefighters, allowing not only the analysis and delineation of the intervention scenarios but also - an important aspect - the support to decision-making for the figures designated to command operations. The TAS is a key function in the reporting phase, which involves the construction of the mapping of the scenario through the information received directly from the emergency scenario. Since the Abruzzo earthquake in 2009, in all disasters, the TAS function has always been activated by the Italian firefighters and has made it possible to draw up thematic maps of significant importance on digital cartography, also from the point of view of the judicial police, as well as operational.

The Italian Fire and Rescue Service has implemented a WebGIS portal, called "Firefighters Geoportal", within which thematic maps (Assets) relating to active emergency scenarios are published. By accessing the Geoportal, it is possible to view the aforementioned thematic maps and the related data, georeferenced and organized on layers. Through the Geoportal it is possible to see the interventions in progress, those closed or pending, see the overall status of the structural assessments of buildings, access through links to more information or to the filming performed by drones (UAS). The main advantages offered by the Geoportal for emergency management are the ability to share data in real time, remote monitoring of events, archiving of geodata by theme and the implementation of maps with data collected in the field and transmitted. Figure 2 shows on the Firefighters Geoportal an emergency that occurred in 2018 in northern Italy, relating to a landslide. The screenshot highlights the contours of the landslide, the buildings evacuated, the forbidden areas, the advanced command posts and the local rescue coordination centers. In the image it is also possible to see that the detachment point of the landslide is close to the area affected by a forest fire, which occurred the previous year and could have indirectly caused it [1]

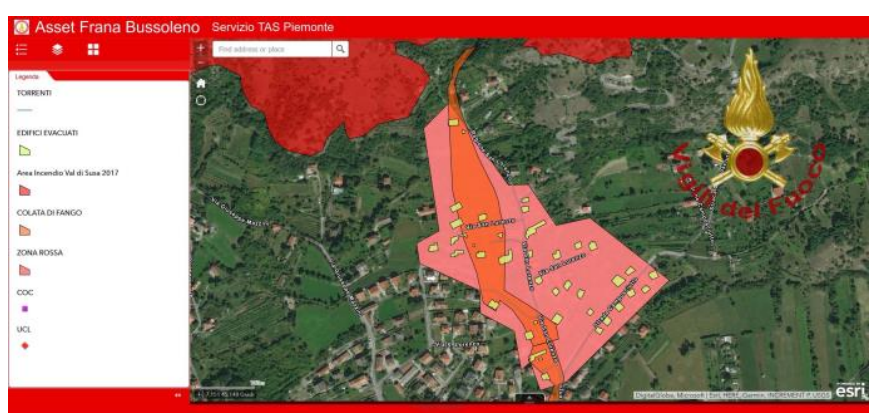

Figure 2. A screenshot of the TAS asset

\subsection{Interoperability and data exchange}

Typically, the need to agree protocols and standards for interoperability between systems of a specific sector is revealed only downstream of the diffusion of new technologies and systems, which individually bring about management improvements, but often result incapable of communicating with each other, generating new and difficult problems. This case also includes interoperability between the systems of operating rooms for the management of rescue: 
until recently, the technological equipment of the operating rooms included telephone, fax, paper, pen and little else. More recently, many operations rooms have equipped themselves with complex computer systems integrated with communication systems, the benefits of which have often been measured in many aspects of rescue management. Unfortunately, the negative sides were not foreseen different systems, proliferated in a competitive market, by producers who had no interest in promoting data exchange with competing systems, then turned out to be unable to exchange data among themselves, that is, to be interoperable.

The Italian Fire and Rescue Service came into contact with the concept of interoperability, thanks to the participation in the European research and development project REACT, which had among its objectives the improvement of data exchange between operating rooms for rescue management. Considering the expected benefits, the Italian Fire and Rescue Service adopted the CAP (Common Alerting Protocol) version 1.1 with two ministerial decrees in 2008 and 2011. The adoption of the communication protocol by decree was the consequence of the decision to open up to data exchange between its own rescue management system and similar systems of the agencies involved in public rescue.

CAP is an XML-based data format for exchanging public warnings and emergencies between alerting technologies, which allows a warning message to be consistently disseminated simultaneously over many warning systems to many applications, such as Google Public Alerts and Cell Broadcast. CAP increases warning effectiveness and simplifies the task of activating a warning for responsible officials. Since the adoption of the CAP protocol, the 100 provincial control rooms, 18 regional control rooms and the National Control Centre of the Italian firefighters exchange a daily average of 25,000 CAP private messages concerning rescue operations in real time. Any emergency stakeholder in Italy which wants to exchange or share data with firefighters during large-scale emergency or rescue operations must adopt the CAP protocol. This greatly promotes the interoperability of technologies and operating rooms of various agencies in Italy and abroad $[1,2]$.

\section{DECISION SUPPORT SYSTEMS}

\subsection{Basic of decision support systems}

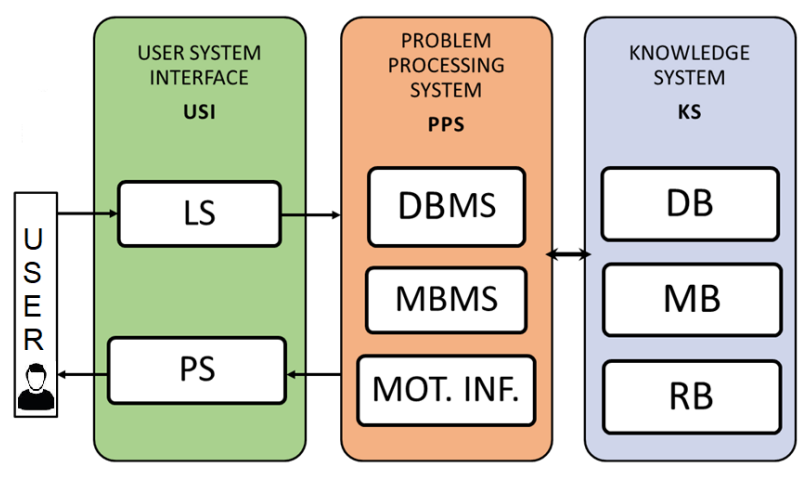

Figure 3. Computerized DSS components

A Decision Support System (DSS) is an interactive computer-based system or subsystem intended to help decision makers use communications technologies, data, documents, knowledge and/or models to identify and solve problems, complete decision process tasks, and make decisions. Decision support system is a general term for any computer application that enhances a person or group's ability to make decisions [3]. Also, decision support systems refer to an academic field of research that involves designing and studying analytical information systems. In general, DSSs are a class of computerized information system that support decision-making activities [4].

A computerized DSS [5] is composed of the following three components (see Figure 3):

(1) Knowledge System (KS), which contains all the information relating to the problem to be addressed

(2) Problem Processing System (PPS), consisting of a processor, which generates useful responses to the process

(3) User - System Interface (USI), which is the main interaction mechanism between user and system

The Knowledge System, in turn, consists of three parts:

(1) Data Base (DB), database, containing quantitative and structured data records, textual information, georeferenced data, images, photos and maps processed by computer

(2) Model Base (MB), model base, containing statistical routines and mathematical/simulation/management models

(3) Rule Base (RB), knowledge base, containing rules and logical relations.

The Problem Processing System, on the other hand, is composed of three parts:

(1) Data - Base Management System (DBMS), which is the management system of the Data - Database

(2) Model - Base Management System (MBMS), which is the management system of the models DB

(3) Inference Engine (MOT. INF.), which is the hardware and software system with artificial intelligence functions.

The User System Interface, finally, consists of two parts:

(1) Language System (LS), interface with the user for data entry and possibly models choice

(2) Presentation System (PS), interface with the user for answers presentation (tables, graphs, maps, etc.)

Even if the concept of decision support was born in the $60 \mathrm{~s}$ and has seen a great evolution already in the $80 \mathrm{~s}$, its application to emergency management is relatively recent. Lately, some European research projects have aimed at identifying new tools and technological resources for the management of multi-agency and cross-border emergencies. One of them, in which the Italian firefighters took part is FIRE IN [6], the aim of this 5-year project is to find capability gaps in fire/emergency/rescue activities in the EU and to suggest promising solutions, addressing the gaps. DSS are one of the promising solutions identified by the EU project FIRE IN. Main advantages in using a DSS are: better assessment of complex scenarios, communication and interoperability improvement, standardization of procedures, time reduction, faster population alert and technical confirmation of the choices made. Moreover, the use of DSS allows to verify emergency plans and to carry out multi-agency exercises.

\subsection{DSS for emergency management}

DSSs are used in the financial services, commerce, telecommunications and healthcare sectors with the aim of 
facilitating the use of data, providing an interactive environment, decision-making support and effectively using models to data analysis.

Only in the emergency sector has their use stopped in research. Projects in this sense that have shown the feasibility of technological solutions have also concerned Italy, such as LIAISON (Location Based Services and Emergency Indoor Location Systems, ECIST 2003), REACT (Control centers and emergency services interoperability, EC-IST 2005), SAVE - ME (Disaster mitigation and evacuation in transportation hubs, EC - SST 2008), IDIRA (Interoperability in large - scale multinational disaster, EC - SEC 2010), AF3 (Advanced Forest Fire Fighting, EC - FP7 - SEC 2013), INPREP (An INtegrated next generation PREParedness program for improving effective inter-organizational response capacity in complex environments of disasters and causes of crises [7], EC - H2020 - SEC - 01 - DRS 2016). These projects have shown that decision support systems can provide real-time event status, and simulation of forest fires, weather events, tsunami waves, floods and provide usable information, together with data from sensors, to make decisions about what people exposed to risk should do.

The purpose of the DSS is therefore to include real-time data and simulation results in the assessment procedures, to substantially improve the safety of people and the efficiency of rescue. The main tools that can be used are:

Sensors:

(1) satellite terrestrial monitoring

(2) video surveillance (forest fires, traffic, structures, etc.)

(3) crowd sensing (position of people, seismic events, etc.)

(4) specific sensors (seismic, hydrographic, etc.)

Simulation:

(1) weather events

(2) flood

(3) forest fires

(4) tsunami waves

(5) diffusion of dangerous substances in the atmosphere

Emergency communication to the public:

(1) television, radio

(2) sms

(3) social networks

(4) internet.

With these tools it becomes possible to switch from a "static" to a "dynamic" Geographic Information System: in other words, it is possible to use cartographic data and alphanumeric data in the context of operational procedures, to monitor in time real activities, procedures and information flows in order to support both the planning and the real-time management of the emergency with greater efficiency. The System, for example, in a computerized and updated cartography can display, on different layers, the critical infrastructures, the houses, the viability, the systems and networks, the signals and requests for assistance, the available resources and movements on the territory, fire and safety devices, information deriving from the same emergency plan and the presence of fragile people, sensitive targets, people with reduced mobility. Furthermore, it can manage the flow of communications between the various operating rooms and the exchange of data and information, also keeping track of all this in a logbook updated in real time. This amount of information is clearly presented in a selective and orderly manner to the decision makers, made up of the top management of the rescue and civil protection organizations, in order to be able to easily and quickly identify the information sought. A DSS, really, is able to do much more, in fact it can simulate using special software, starting from the data entered also in real time and provided by sensors (for example weather stations and seismographs) and by rescue teams, consequences on the population and on the territory of an emergency event, presenting them on the aforementioned cartography in the form of risk areas based on threshold values taken from national and international regulations, but also definable by users. Likewise, it may also be able to simulate the evacuation of some areas, calculating the times and evaluating the feasibility of this operation.

An application of DSS, based on Machine Learning, which could in the future be implemented by the Italian Fire Brigade relates to the possibility of automatically labeling an intervention as relevant, or as not relevant, to be able to warn the competent authorities and better manage the event, avoiding both bureaucratic and political problems. Currently the Fire Brigade uses a deterministic system based on a system of fixed parameters, which tries to capture all the characteristics that an intervention must have to be classified as relevant, but this approach often leads to an excessive generation of false positives. Therefore, the main challenge to be faced concerns the very definition of relevant intervention, i.e., what characteristics an intervention must have to be defined as relevant. To implement such a system, it is necessary first of all to use a correct set of intervention data, with the classifications as relevant or not relevant (based on the reports received) and then establish a series of other relevant parameters (features), such as the number of updates, the overall duration of the operations, but also the description of the characteristics of the territory from the GPS coordinates (to understand if you are in a built-up area, an industrial area or in an inaccessible area).

The objective of using DSS, in this case, is to "automate" and therefore speed up the response of the structures involved in an emergency, leading to a significant reduction in intervention times by the structures and subjects who must take action in case of need, as well as an increase in the response efficiency in qualitative terms.

\subsection{Technical features of an emergency management DSS}

From the user's point of view, the main characteristics that a DSS of this type must possess are:

(1) to be as feasible and platform independent as possible.

(2) to be easy to use and user-friendly to users with intermediate computer skills.

A valid and tested technological architecture for these systems is the client-server one, because it ensures the division of the application operation logical model into smaller functional units [7]. The user interface can be easily based on a web platform and this choice offers the following advantages: (1) the communication protocol has already been implemented and tested on an international scale (HTTP/HTTPS).

(2) at the client level, no specialized software is required, only a web browser is needed, so the architecture can be implemented on many systems, without particular configuration requirements.

(3) system upgrading can be a simple operation.

(4) data transmission security is ensured by using the HTTPS, which provides a secure channel between the client station and the system residing on the server. 
On the client-side, as mentioned, a web browser and an internet/intranet connection are required, while on the server side, at least:

(1) an Operation System, for example Linux Based, which has good stability both in terms of security and performance.

(2) a software for managing the various system components, written in a programming language that allows effective interaction and data exchange.

(3) a DBMS, that is the software system that enables users to define, create, maintain, and control access to databases [8], which had already been mentioned.

(4) a web server, that is a software and underlying hardware that accepts requests via HTTP, the network protocol created to distribute web pages.

\subsection{DSSs and emergency planning}

Many emergency plans on a territorial scale seem to have been created in analogy to those that are prepared for buildings, despite the great difference between the realistically possible scenarios. For buildings, information is known on what to do in the event of a well-known event (i.e., a fire), in a context already planned for the exodus, with a well-known occupancy and crowding, a partially predictable scenario and fire detection and alarm systems specially designed which the occupants are familiar with, but very little of this can be foreseen on a territorial scale. The fundamental variables for planning with accuracy are too many to solve the problem of managing the exodus phases a priori. Furthermore, the infrastructures have rarely been designed according to risks and equally rarely according to the exodus, with numbers of people involved and much more onerous situations and risks to be foreseen. A traditional planning, which defines in advance what to do during the emergency and foresees what information to give to the people involved, should consider a number of scenarios equal to the risks multiplied by the number of areas that can be affected and by the number of different conditions (night, day, summer, winter, weather conditions, etc.), which affect the distribution of people and the availability of rescuers. The related evacuation plans, however accurate, could never be as realistic as those assessed on the situation existing in the emergency scenario. A valid reference for identifying the potential risks to which the population may be exposed, which the plan plans to secure in the event of an emergency is offered by Chapter 5 of the NFPA 1600 standard (2020 edition). It divides risks into categories, including geological, meteorological, biological, human unintentional (including industrial risk and conventional CBRN risk), intentional human (including the risk of terrorist attacks), IT, economic - financial and strategic. Starting from this reference, it is possible to understand how great the number of risks and credible scenarios can be for a typical territory and therefore how demanding the task of planning for the emergency becomes. The plans prepared to manage emergencies at a territorial level are usually conceived to proceed in an organized way with the deployment of rescue and civil protection resources. A civil protection emergency plan answers questions like this: where are the base camps located? Which entities should be involved? Who has to coordinate locally? Where are the command and control centers established?

The Italian guidelines on civil protection planning provides that each plan must include these three parts:
(1) General part, inherent to the characteristics of the territory concerned.

(2) Outlines of planning, in which response objectives and competences are set by the various agencies.

(3) Intervention model, in which decision-making responsibilities are assigned, a chain of command and criteria are established for a rational use of resources and communications.

The plan, of course, must be constantly updated both because of the evolution of territory and population, and because of the feedback from the exercises.

A further and important functionality, which can be available in a DSS used for prevention and rescue purposes, is precisely that of carrying out simulations of emergency scenarios, which can be studied, or used directly to carry out protection and civil defense exercises, multi-agency, both multi-agency and with partial deployment of resources on the territory. The simulation allows, in this case, to verify the effectiveness of some choices made during the drafting of the plan and to appropriately allocate the available resources, always having an overview of the situation (thanks to the Geographic Information System) constantly updated.

Furthermore, being able to manage information flows and, in some cases, also communication tools, a DSS can allow, in a short time, to send alerts to the population. Some examples of the aforementioned tools are notices on motorway and road panels, the sending of text messages and the use of messaging systems and the use of special mobile applications to provide information in the event of an emergency. More particularly, the use of technological platforms of the type of Decision Support System, i.e., decision aid systems, can serve to immediately direct information to people exposed to risks.

\subsection{Evacuation simulation and DSSs}

During an emergency there can easily be a need to evacuate people from an area. When planning an evacuation, it is essential for civil protection authorities to know which areas will be affected by the event (for example a forest fire, a flood, a dangerous concentration of a toxic substance, etc.), when the areas will become untenable, how long it will take occupants in these regions to evacuate and how long it will take occupants to reach a designated place of safety.

Urban scale agent-based evacuation modeling tools offer the potential to address all these issues, especially if they can be integrated with emergency management DSS. In agentbased modeling (ABM), a system is modeled as a collection of autonomous decision-making entities called agents. Each agent individually assesses its situation and makes decisions on the basis of a set of rules.

The EXODUS large scale evacuation modelling system is an agent-based evacuation model capable of simulating the evacuation of large populations, measured in the hundreds of thousands, in large scale environments, as many square kilometers. It is based on the popular buildingEXODUS $[8,9]$ software and consists of three components: three parts:

(1) urbanEXODUS, a desktop interface, which was developed to enable the EXODUS engine to easily represent large scale urban space.

(2) webEXODUS, a portable GIS based web interface, which was developed to be utilized during an incident.

(3) the EXODUS engine, which receives simulation inputs from urbanEXODUS or webEXODUS, performs the simulations and returns the results. 
EXODUS can simulate an evacuation on foot of an area, but to have a realistic simulation it is necessary to consider also evacuation by car. A possible solution is the software "Simulation of Urban Mobility" (SUMO), which is an open source, highly portable, microscopic and continuous traffic simulation package, designed to handle large networks. It allows for intermodal simulation including pedestrians [10].

The integration of the two software models therefore allows realistically simulating an evacuation and as we will see it can be used by a DSS.

\subsection{Application of a DSS}

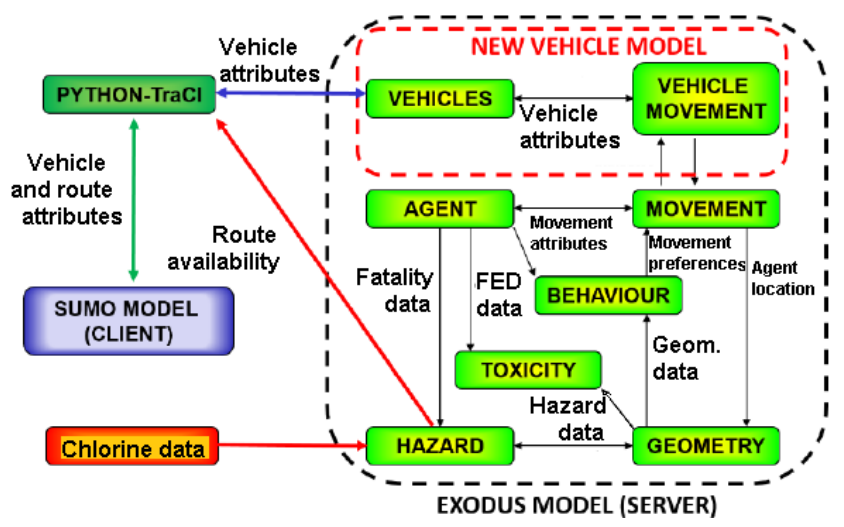

Figure 4. EXODUS, SUMO and hazard models integration

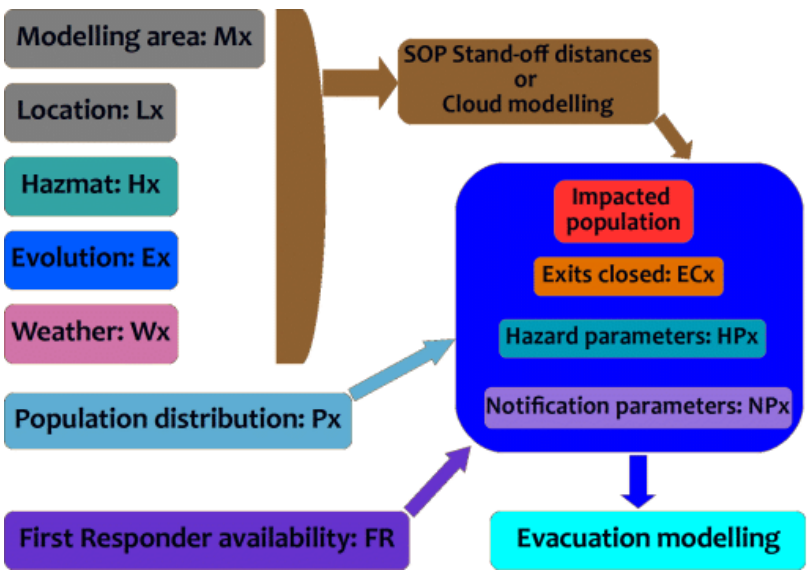

Figure 5. The process/architecture of the DSS adopted in the exercises of the IN-PREP project

A DSS for emergency management has been used during the exercises of the IN-PREP EU project. The project held its $2^{\text {nd }}$ full-scale exercise (FSX) in Genova and Savona, Italy on 28 October 2020. This was the penultimate full-scale exercise of the IN-PREP Project and the first to be carried out remotely due to the Covid-19 pandemic. The $2^{\text {nd }}$ FSX of the project simulated the response to a motorway incident involving a chlorine chemical spill, in the Italian region of Liguria affecting mainly the municipalities of Vado, Quilano and Savona. The analysis of the evacuation scenarios provides a quantitative assessment of the impact of different evacuation procedures on the evacuation performance including the population's likely hazmat exposure [11, 12]. During the exercise were used EXODUS as pedestrians' evacuation simulation model and the well validated SUMO vehicle movements simulation model. Figure 4 shows the integration of EXODUS, SUMO and hazard models in the DSS. The scenario started from a single non-intentional incident: a truck carrying hazardous material (Chlorine) has an accident and starts losing its contents within an urban area. The exercise was carried out using the resources realistically available at specific dates and hours. The DSS used was fed with real/realistic data, taking into account the possibility that the emergency managers could realistically access such data in the time available.

The diagram in Figure 5 shows the main parameters and data considered by the DSS and how they interact with each other $[11,12]$.

(1) Modeling area: the definition of the area to be modeled within the EXODUS evacuation tool it represents a tradeoff between the accuracy of the model and the commitment of computers in terms of time and resources to build it. The area considered is inhabited by 22,981 people, divided between the three municipalities of Vado, Quilano and Savona.

(2) Location: is where the accident occurred, in the municipality of Vado.

(3) Hazmat: Chlorine was used because it is a toxic substance which does not ignite, but remains buoyant harming people in its path.

(4) Evolution: three release quantities and durations for Chlorine (450 kg, $810 \mathrm{~kg}$ and $216 \mathrm{~kg}$ ).

(5) Weather and Population distribution: The incident was assumed to take place in October 2020, with good weather conditions, light wind and no rain. Weather forecasts over 6-8 hours after the event time were calculated through the U.S. National Oceanic and Atmospheric Administration HYSPLIT [13] web app.

(6) SOP Stand-off distances or Cloud modeling: on the basis of the scenario and the parameters introduced, the system identifies the evolution of the chlorine cloud and the distances from the accident below which irreversible and reversible injuries can occur. The distances were derived from the WISER app and software [14].

(7) First responder availability: a realistic first responder availability as a function of date and hour for the selected days was considered, including roles, activation sequence and distance from their base.

(8) Exit closed: the system considers the main communication routes exiting the places to be evacuated, the information provided to the population and both the spontaneous behavior of people aware of the danger as well as the impact on them of the instructions provided.

(9) Hazard parameters: the evacuation model allowed for the calculation of time-distribution of the exposure of the population to risk of the potential impact on health in case of chlorine propagation. To do this, the model received as input the scenario-depending time series of isoconcentration shapes based on two toxic threshold concentrations: AEGL-1 and AEGL-2 [15].

(10) Simulation stop times: for each scenario the evacuation simulation ended at the first-time step where the hazard parameters fell below the previously mentioned thresholds.

(11) Notification parameters: For the exercise the notification methods considered included: the most common soon after the event, based on officers' direct alert, by ringing the intercom, and automated Phone Call systems (or texts).

In Figure 6, an image provided by the software is shown: there are fifteen "alert zones" (areas to be eventually evacuated) in three municipalities which are Vado Ligure 
(orange), Savona (yellow) and Quiliano (dark grey) and the light grey cloud shows the trend of the chlorine gas dispersion. The red circles on the map indicate shelter/refuge locations for people evacuating by foot and the red stars are the exit points from the region for people using vehicles. During the exercise, the DSS simulated six scenarios, related to six different strategies, evaluating their outcomes to assist authorities to make well-informed decision. The six scenarios were: no kind of instructions given to the population, all people shelter-inplace, all people evacuate, two, four and seven zones shelterin-place. The simulation showed that the third scenario (all evacuate) has the highest exposure levels, the first and second scenario have the lowest exposure levels and generally the more zones that are notified to shelter the lesser the exposure.

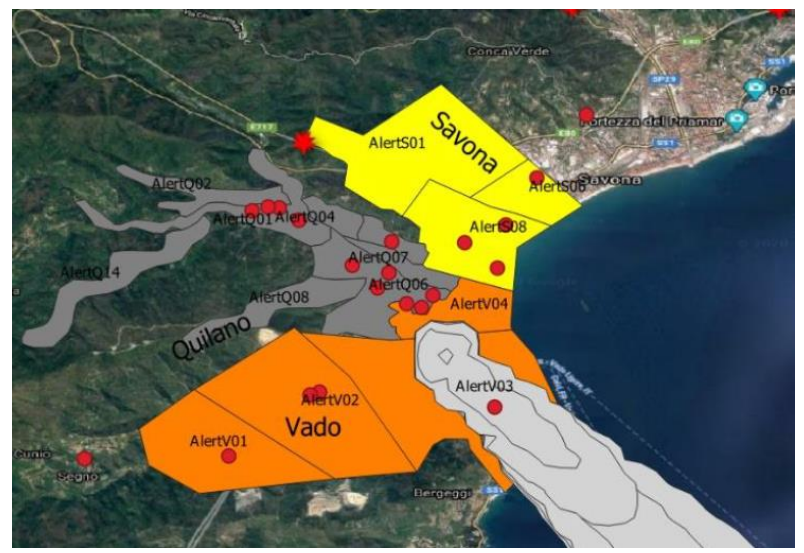

Figure 6. Screenshot of IN PREP DSS, during the $2^{\text {nd }}$ FSX

\subsection{Criticality in the use of DSS}

The use of DSS with support functions in emergency management certainly shows positive aspects, as we have seen in the previous paragraphs, but it, however powerful it may be, can only be useful if the emergency management policy provides that:

(1) the authorities in charge of emergency management integrate in the emergency plans the elements for assessing the situation in real time (evolution of a fire, propagation of a toxic cloud, flooding, etc.) that the DSS provide.

(2) citizens are adequately informed on the greater management capabilities that the platform allows and, on the channels, and methods of communication in emergencies.

However, also in view of the findings on the use of these systems, obtained in Italy and Europe, some factors have emerged that can make their effective use difficult by the emergency managers [12]. The main problems encountered may be summarized in the following points:

(1) the cost and complexity of systems that are able to keep the evolution of the event under observation (from the moment it arises for the entire duration of the emergency, for example, forest fire, flood, release of toxic effluents into the atmosphere), factors that have slowed down the demand for the design of integrated DSS.

(2) the ability to assess on an objective basis whether the time of departure is adequate to save people. What is normally done in fire safety (the comparison between the time available for the exodus and the time needed to escape) is only now beginning to be applicable to larger scales. The main obstacle is the availability of simulation applications for the exodus on a territorial scale, starting from updated data on the position of people (i.e., central areas of cities are much more crowded during the day than at night, tourist areas are more crowded in summer, etc.).

(3) the difficulty of using information channels that reach the entire population. An example of such communication problems is the impromptu use of social networks. The alarm should reach all citizens, regardless of the tools they use to be informed (an issue that concerns the digital divide present in all societies). Also in this case, while considering that informing one hundred percent of citizens in real time is an unrealistic goal, the systems and protocols capable of integrating the various means of communication (media) are only now starting to be disseminated.

(4) the lack of a unified data exchange protocol. The costs of creating a sensor network could be considerably contained if the DSS could use data from devices present in the area. The role of the data exchange.

(5) protocol is to allow different systems to communicate without changes for the systems themselves. Unfortunately, adherence to unified protocols (in the emergency sector the most used protocol is the CAP Common Alerting Protocol, described before) occurs only on a consensual basis. This among the problems is one of the most relevant, as the adoption of a single and widely shared and tested protocol among the various entities would allow to greatly streamline the complexity of the system.

(6) the difficulty in finding new professionals capable of designing and managing systems that involve such different disciplines (emergency management, data exchange technologies, sensors, communications and risk assessment), which however could be solved in the future, with the development of the industry.

\section{COMMUNICATION SYSTEMS}

\subsection{Telecommunications of the Italian firefighters}

There is a clear link between DSS and communications systems, as both are based on information and communications technologies (ICT) and are often integrated with each other.

In fact, the three basic objectives for achieving success during an emergency are:

(1) coordination, understood as the unitary direction of operations, through a global vision of the intervention.

(2) the management of resources, through a rational and timely use of the men and suitable means available for emergency operations.

(3) communication, which in turn must be distinguished in external communication, directed to the population and the mass media, and internal communication, relating to the exchange of information between the parties involved in the management of the emergency both locally and centrally.

The two key aspects of internal communication in emergencies are the instrumental resources to be used, so that they allow the rapid and flexible exchange of information in any scenario, and the use of a unique, clear, immediate and shared language by all operators. The instrumental resources here are Mission Critical Systems: to have your own 
communication system allows you not to depend on other connection systems (e.g., public networks), which may not be usable right in the moment of a serious emergency.

The instrumental resources for emergency communications of the Italian Firefighters are:

(1) its own analogue Private Mobile Radio network based on simulcast channels, operating on special frequencies assigned by the Ministry of Defense, which allows connections throughout the national territory.

(2) narrow band applications for voice and data communications, geolocation, short coded and free messages: DMR technology (being implemented) and TETRA technology (for advanced command posts and teams).

(3) its own satellite network mainly used for major emergencies.

The analogue radio communication networks of the Italian firefighters fall into the type of SIMULCAST (Simultaneous Broadcast) networks, also known as synchronous isofrequency networks. These, as the word itself says, provide to the radio coverage of the service area using different repeaters all operating at the same frequency and working in a coordinated manner, to the broadcasting of the signal to the mobile terminals [1]. This radio network is structured on various levels:

(1) Local Networks, for communications between Commands, smaller headquarters and vehicles.

(2) Regional Networks: for communications between Regional Departments and Local Commands.

(3) National Network: for communications between National Operations Center, Regional Directions and Provincial Commands. The national interconnection network is divided into four subnets: North - East, North - West, South and West sub-networks, connected to the regional and local simulcast networks.

DMR (Digital Mobile Radio) is an open digital radio standard, developed by ETSI (European Telecommunications Standards Institute) for professional mobile radio communications (PMR) equipped with advanced voice and data functions [16]. The analog SIMULCAST network will be replaced soon by one based on DMR technology, characterized by better performance in terms of number of channels [17] and therefore of simultaneous communications available, consistent performance on the coverage area and simultaneous voice and data transmission.

The TETRA (Terrestrial Trunked Radio) system is a technology of digital radio wave communication designed specifically for applications in the field of professional private radio networks. TETRA was created with the aim of combining the merits of modern cellular radio technologies with the reliability and versatility of old analogue technologies. It is characterized by an automatic assignment of the available transmission channels in a dynamic way [18].

TETRA technology is used by firefighters to communicate on complex emergency scenarios, as it allows communications (private, group and broadcast) between teams, even from different agencies and advanced command posts, with the sending of data and images.

The satellite network of the Italian firefighters is part of the Satellite Backbone, that is a reliable communications system for emergencies, supplied to the Italian Fire and Rescue Service and the Civil Protection Department, capable of conveying any type of communication originating from fixed or mobile devices between the Operations Room and the territory. Each user has his own subnet, which by interoperating with the Backbone creates a TLC infrastructure independent from public networks and managed independently. The system is designed to meet the needs of Italian Institutional Agencies in charge of emergency management [19].

\subsection{A resource for rescuers: LTE mobile cells}

Among the broadband communication systems that can be used for the Italian Firefighters communications, there is particular attention to the LTE system, and this arises from the need to have available, during an emergency, new technologies suitable for the transmission of images, videos and data. The infrastructure necessary for a proprietary network is however extremely heavy in terms of costs, sites, equipment, power systems which make it almost impossible for a single entity to build a proprietary network. Instead, it is conceivable to use infrastructures built by public managers with priority access to be allocated to rescue. The release of the LTE standard currently being defined provides for "mission critical" communication with the use of PTT (Push to talk) also in direct communication (Direct Mode) and communications carried out with terminal devices capable of repeating the remote signal. These performances would release the user from the presence of network coverage.

The market offers some field-type products capable of creating communication "bubbles" in critical areas, such as the Rapid eLte Emergency Solution, a device capable of immediately creating an LTE and $3 \mathrm{G}$ network with Wi-Fi support, in order to quickly enable the activation of an emergency mobile network. This is a mobile communication infrastructure that guarantees extremely fast assembly and installation times, and allows the transmission of voice and data via the mobile network, especially images and videos, even in HD. The system makes a joint use of different radio frequencies to acquire images of the disaster scenario and then send them to the operations room in video streaming. Technically it is a trunking solution equipped for extreme situations and including a base station, broadband eLte that allows voice and video dispatching and is based on an advanced connection technology that can involve satellite, mobile or wired networks. The device provides a transfer rate of $100 \mathrm{Mbit} / \mathrm{s}$ in the downlink and $50 \mathrm{Mbit} / \mathrm{s}$ in the uplink. In this way, a single network can support multimedia exchanges, voice and video scheduling, high-definition video surveillance, ultra-remote data acquisition and mobile office services. With the Rapid eLte Emergency Solution, you can set up a wireless network in 15 minutes in any type of emergency. The preconfigured components allow use even by non-technical personnel and are available for protected transport both on the vehicle, thanks to the special suitcase (see Figure 7), and on foot in the "backpack" configuration [20].
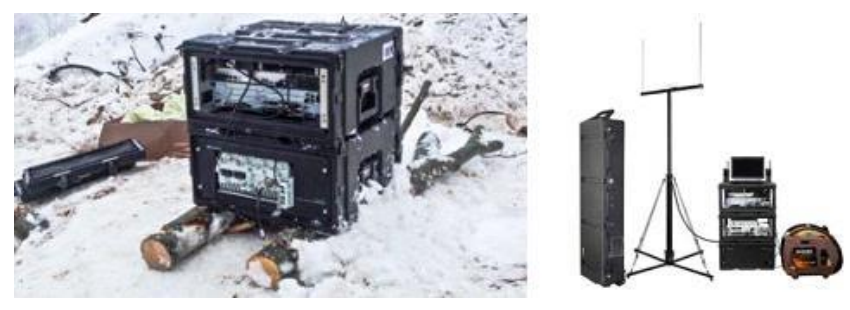

Figure 7. Suitcase (left) and components (right) of the Rapid eLte Emergency Solution 
This solution was used for the first time by the Italian Firefighters during the emergency caused by the avalanche that hit the town of Rigopiano in 2017, to ensure timely communication between the rescuers present in the hotel and the control center from which the emergency was managed. The Rigopiano avalanche was an event that occurred on 18 January 2017 at the homonymous locality located in the municipality of Farindola, in Abruzzo. The avalanche, detached from a mountain ridge above, hit the Rigopiano-Gran Sasso Resort hotel, causing 29 victims. On the basis of the available data, this is probably the avalanche, which in Europe caused the greatest number of deaths after 1999 .

Once on the scenario, the LTE bubble allowed all operators to be connected and to be able to transmit the images from inside the Rigopiano hotel to the operations room, many of the rescue images seen on TV came from this system. The event represents a breaking point: before, operators could only communicate by voice in push-to-talk (PTT) mode, today, with this telephone carrier condensed into two suitcases, they can talk and see the images transmitted by the devices supplied to USAR operators. In this way, from voice communication to live multimedia, rescuers are relieved of the task of describing (in a subjective way) the situation. Basically, the rescuers had an active mobile communication infrastructure available not only on the surface, but also underground, having in fact also inserted an antenna in the tunnels that had been dug, to receive images from the inside. This allowed the rescuers to directly transmit voice and images, sharing information about the disaster with the operations room. It was thus possible to make decisions taken remotely after assessing the risks for the rescuers according to what was visible on the video, in relation to the internal structure of the hotel moved by the avalanche about ten meters from the original position [1].

The evolution of this tool could lead in the future to simultaneously connect the various rescuers (law enforcement, firefighters and civil protection), who use different frequencies in the transmission of information during normal rescue activities. The goal is to have timely coordination of emergencies between the various operating rooms in the idea of a smart city.

\subsection{The Dedalo system for search and rescue operations}

During the search for missing people, it has been discovered that, among the personal items, there is very often a mobile device (cell phone) unusable to ask for help in areas which are not covered by commercial operators. Italy has a particularly heterogeneous orography: more than half of the territory is made up of rural or semi-rural areas (for example mountainous or hilly areas), which are isolated and sparsely populated. These zones are often devoid of the necessary infrastructure for mobile coverage. The Dedalo system (see Figure 8), in the absence of signal from mobile phone operators, allows to create a temporary GSM infrastructure suitable for: registering commercial cell phones, estimating the position of the source and allowing the location by the rescue team [20].

Also, on the Rigopiano disaster, this new instrumentation was used, because Dedalo is a system that improves the search for missing persons, especially in inaccessible areas and without telephone coverage. The system made it possible to find and rescue nine people trapped under the rubble. In fact, it allows rescuers to locate a mobile phone even in the absence of normal telephone coverage, creating a GSM emergency network, which allows both to locate the equipment of a possible missing person and to establish a voice and messaging link [1].

Now the system is in the final phase of experimentation by the Italian Firefighters, in order to be used in national and international disasters.

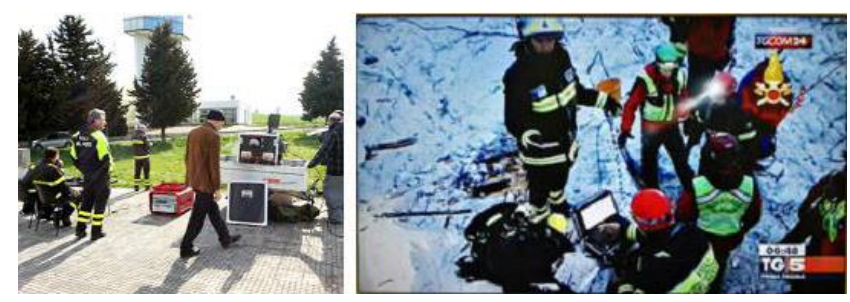

Figure 8. Use of the Dedalo system during an exercise (left) and to search for missing persons in a rescue operation (right)

\section{RELIABILITY OF EMERGENCY ICT SYSTEMS}

In general reliability is defined as the probability that a given system or component will operate without any failure for a given time when used under specified environmental condition. For ICT systems a distinction must be made between:

(1) hardware reliability

(2) software reliability

(3) quality of the data provided

The first two relate to the ability of an ICT system to perform its function without interruption and the third is linked to the system's ability to produce output data that meet the requirements set by users. An output that does not meet the requirements could be, for example, an incomprehensible conversation, or an imprecise geographical location. The DSS for emergency management and the ICT systems for emergency communication are called "mission critical", in the sense that they are essential for the organization and that their malfunction has a significant impact on the activities of the organization itself. An additional difficulty is constituted by the fact that ICT systems for emergencies must work in realtime, responding immediately to user requests and changes in the scenario.

The reliability of the hardware, however complex, can be traced back to that of electronic devices and can be increased with the use of redundant systems. For the software it is different. Mission critical software are vastly different from normal personal computer programs. In many situations, failure of this kind of software results in loss of life, therefore failure is not an option for them and in the odd situation that they do fail, there must be back up measures in place to prevent catastrophe. This is the reason why, to increase reliability, the software engineering must include the following functions: fault prevention, fault detection and fault reaction.

This software usually run on multiprocessor machines, which can perform multiple computations at the same time. Parallel processing is necessary in real-time systems because the nature of the real world is such that various data are requested at the same time. When multiple processes are executed simultaneously, the system must manage the resources available in terms of computing power and memory between them, preventing two different processes from competing for the same resource, which would lead to failure. Software faults can be permanent, transient, or recurrent; the first occur and persist until the cause is repaired, the latter 
occur, persist for a time, then disappear and the third occasionally repeat themselves. A system fault may instead consist in an interruption of operation, in providing correct data but not at the requested time or in providing wrong data. While in the first case the cause can be both hardware and software, in the second and third the cause will depend on the software and there will also be output quality problems. To deal with software faults which can lead to failure there are two approaches: fault prevention and fault tolerance. The best results in terms of reliability are obtained by using both. The first approach is characterized by an accurate software design, by a careful selection of the input data and by an intense testing before use. The second approach foresees that in the presence of errors the system works with limitations, resets itself by restarting, or maintains full functionality even if for a limited time. This latter case represents the goal towards which mission critical software aims [21].

To prevent a system, such as a DSS, from supplying wrong data, it is necessary to foresee both the presence of output verification algorithms and the possibility of reaching the same results with different models and comparing the data provided by them. Essentially there must be a voting algorithm capable of evaluating the reliability of the output by comparing it with forecasts, probabilistic distributions and historical data [22].

In addition to internal errors, ICT systems for emergencies must also be protected from external threats. Cyber-attacks are becoming more frequent and can be launched for various reasons. The subtraction of personal data from large databases, such as those mentioned above, can be a source of income for cybercriminals and a very widespread attack is precisely that of the ransomware type, in which data is encrypted and to decrypt it is asked for a ransom. Another frequent attack is that of the denial-of-service type, which floods systems, servers, or networks with traffic to exhaust resources and bandwidth. As a result, the system is unable to fulfill legitimate requests.

Against cyber-attacks it is necessary to use all available hardware and software resources, but also to develop and maintain an IT culture in those who use the systems, aimed at maintaining cybersecurity standards. In fact, recently both private companies and public bodies have been subjected to phishing attacks, in which false communications are sent, which appear to come from a reputable source, usually through email, to steal sensitive data, as passwords.

In summary, although the bodies that deal with emergency management are often public institutions, with ICT systems well protected from external threats, it is evident that the reliability of these systems depends on many factors, none of which can be overlooked.

\section{CONCLUSION}

The management of an emergency is configured as a complex operation and this is precisely the main difficulty that the various subjects involved encounter, both in the planning phase and in the operational phase. The complexity consists in the need to integrate and coordinate the actions of many and different actors, bearers of differentiated skills and knowledge, all necessary to overcome the emergency in progress. In response to this need, the search for modern, computerized systems is born, interconnected with communication networks and sources of information, which allow effective interoperability between the bodies in charge of emergency management and rescue, and which allow optimization of the time and resources available, achieving the result of providing a quick, timely, widespread, but above all effective and efficient response to the population involved. As we have seen, examples of these systems are the software that operates as Decision Support Systems and the monitoring, control and alert systems, but the technology in this sector is rapidly evolving and there could be many new solutions available to future emergency managers.

In this sense, the recent evolution of the Italian legislation relating to Emergency Planning, both at the central level and at that of the Local Authorities, has allowed a modernization of emergency management methodologies. As mentioned, it is possible to implement and partly verify the functionality of a Civil Protection Plan, using the most modern resources provided by ICT, which can achieve a more efficient management of Civil Protection events.

The objective of this approach is to "automate" and therefore speed up the response of the structures involved in an emergency situation, leading to a significant reduction in intervention times by the structures and individuals. that must be activated in case of need, as well as to an increase in the response efficiency in qualitative terms. An important resource for the purpose of this automation is the Artificial Intelligence (AI), whose implementation is foreseen in the context of DSS. With the development of the computerization process, an enormous amount of data is accumulating from various platforms, which, however, are difficult to interpret, if not correctly highlighted and crossed to control the processes. To this end, one of the branches of AI that is particularly effective and endowed with great potential is Machine Learning (ML): the ability of machines, computers and robots, to learn from experience and then act independently, without any specific programming.

However, the management of emergencies, carried out both with the technologies mentioned and with traditional tools, can never be separated from an optimal management of internal and external communication. The close link between emergency management and communications is evident and is underlined by all situations, in which suboptimal communication has inevitably led to delays and inefficiencies in rescue operations. Also, for this reason, certainly, information technology, which represents a key element of management and decision support systems, is increasingly present in the world of telecommunications, to the point that the two subjects are often not mentioned individually, but combined in the term ICT.

Recent developments in this area, as well as being new resources for rescue, which today make possible planning, responses, results and resource management that were unthinkable yesterday, present us with new and important challenges for our future. Referring only to the most current of these challenges, in line with the development of the technologies used, the need to implement a correct and adequate management of cybersecurity immediately emerged, focusing attention on three aspects: the availability of data (therefore guarantee of access and usability), the protection of information assets and personal data (guaranteeing integrity, designing reliable and redundant systems and ensuring that the information does not undergo changes and cancellations as a result of errors, or voluntary actions, or as a result of malfunctions of technological systems) and privacy, i.e. security management such as to minimize and mitigate the risks related to the access and use of information in an 
unauthorized form. It is therefore clear that those involved in rescue and civil protection, even in major emergencies, only by managing these challenges, will be able to make full use of all the advantages that technological development brings us in this area.

\section{REFERENCES}

[1] Italian Fire and rescue Service. https://www.vigilfuoco.it, accessed on Nov. 9, 2020.

[2] OASIS (Organization for the Advancement of Structured Information Standards) Emergency Management Technical Committee. https://www.oasisopen.org/committees/emergency/, accessed on Jan. 4, 2021.

[3] Keen, P.G. (1978). Decision Support Systems: An Organizational Perspective, Addison-Wesley, Boston, Massachusetts, USA.

[4] Power, D.J. (2001). Supporting decision-makers: An expanded framework. Informing Science, 1: 1901-1915.

[5] Bonczek, R.H., Holsapple, C.W., Whinston A.B. (1981). Foundations of Decision Support Systems. Academic Press. https://doi.org/10.1016/C2013-0-10396-0

[6] FIRE-IN European project website. https://fire-in.eu/, accessed on Jan. 4, 2021.

[7] Daconta, M.C., Obrst, L.J., Smith, K.T. (2003). The Semantic Web, John Wiley \& Sons

[8] Connolly, T.M., Begg, C.E. (2014). Database Systems A Practical Approach to Design Implementation and Management (6th ed.). Pearson. ISBN 978-1292061184

[9] Chooramun, N., Lawrence, P.J., Galea, E.R. (2012). An agent based evacuation model utilising hybrid space discretisation. Safety Science, 50(8): 1685-1694. http://dx.doi.org/10.1016/j.ssci.2011.12.022

[10] SUMO, Simulation of Urban Mobility, http://sumo.dlr.de/userdoc, accessed on Jan. 4, 2021.

[11] IN-PREP European project website. https://www.inprep.eu/, accessed on Jan. 4, 2021.

[12] Marsella, S., Pozzi, D. (2019). Evacuation Planning as a Key Factor in Disaster Management: The contribution of the H2020 IN-PREP action. Proceedings of the 10th International Multi-Conference on Complexity, Informatics and Cybernetics (IMCIC 2019).

[13] National Oceanic and Atmospheric Administration HYSPLIT. https:/www.arl.noaa.gov/hysplit/hysplit/, accessed on Nov. 9, 2020.

[14] US National Institute of Health - National Library of Medicine - Wireless Information System for Emergency Responders (WISER). https://wiser.nlm.nih.gov/, accessed on Nov. 9, 2020.

[15] US EPA Acute Exposure Guideline Levels for Airborne Chemicals. https://www.epa.gov/aegl/, accessed on Nov. 9, 2020.

[16] European Telecommunications Standards Institute. https://www.etsi.org/, accessed on Jan. 4, 2021.

[17] Ulema, M. (2019). Fundamentals of Public Safety Networks and Critical Communications Systems: Technologies, Deployment, and Management. WileyIEEE Press, Piscataway, New Jersey, USA, 107-120. https://doi.org/10.1002/9781119369554.ch7
[18] Cancellaro, M. (2010). Multimedia communications over TETRA 2 networks. Ph.D. dissertation. Department of Applied Electronics. Roma Tre University, Rome, Italy

[19] Telespazio S.p.A. https://www.telespazio.com/, accessed on Jan. 4, 2021.

[20] MPM Telecommunication S.r.l. http://www.mpm-tlc.it/, accessed on Jan. 4, 2021.

[21] Burns, A., Wellings, A. (2009). Real-Time Systems and Programming Languages. Addison-Wesley.

[22] Ionescu T.B. (2013). Reliability of decision support systems for nuclear emergency management. Dissertation. Universität Stuttgart, Fakultät Energie, Verfahrens und Biotechnik. IKE, Bericht, 4-159. http://dx.doi.org/10.18419/opus-2156

\section{NOMENCLATURE}

AEGL Acute Exposure Guideline Levels for Airborne Chemicals

AI Artificial Intelligence

ABM Agent Based Model

CAP Common Alerting Protocol

DB Data Base

DMR Digital Mobile Radio

DBMS Data Base Management System

DSS Decision Support System

FSX Full-Scale Exercise

GIS Geographic Information System

GSM Global System for Mobile Comms.

HD High Definition

ICS Incident Command System

ICT

IT

KS

LS

LTE

$\mathrm{MB}$

MBMS

ML

MOT. INF.

Information \& Communication Technology Information Technology

Knowledge System

Language System

Long Term Evolution

Model Data Base

NFPA

NIIMS

Model Data Base Management System

Machine Learning

Inference Engine

National Fire Protection Association

National Interagency Incident

Management System

PMR Professional Mobile Radio

PPS Problem Processing System

PS Presentation System

PTT Push-to-talk

RB Rules Data Base

SDSS Spatial Decision Support System

SOP Standard Operating Procedures

TAS Topography Applied to Rescue

TETRA Terrestrial Trunked Radio

TLC Telecommunications

USAR Urban Search And Rescue

UAS Unmanned Aerial Systems

USI User System Interface

XML Extensible Markup Language 